Palabra Clave (La Plata), abril 2017, vol. 6, n² 2, e022. ISSN 1853-9912

Universidad Nacional de La Plata.

Facultad de Humanidades y Ciencias de la Educación.

Departamento de Bibliotecología

\title{
Diagnóstico de los sistemas de información en las empresas priorizadas según los requerimientos actuales
}

\author{
The state of the systems of information in the \\ companies prioritized according to the current \\ requirements
}

\section{Grisel Castillo Almeida $*$, Elio Moisés Pérez Rodríguez **}

* Centro de información y gestión tecnológica, CIGET Pinar del Río, ** Universidad de Pinar del Río Hermanos Saíz Montes de Oca, Cuba | gcastillo@ciget.vega.inf.cu,

eliop60@upr.edu.cu

\section{PALABRAS CLAVE RESUMEN}

Sistemas de información

La necesidad de mantener sistemas de información eficientes en las empresas priorizadas del territorio de Pinar del Río (Cuba), nos llevó a realizar un diagnóstico para determinar el estado actual de los mismos y proponer acciones de conjunto que faciliten la toma de decisiones y la identificación de los flujos de información útiles, para favorecer el desempeño organizacional. Los resultados del estudio arrojaron, entre otros datos, que solo el $12 \%$ tiene un sistema de información organizado e implementado según los requerimientos actuales, lo que indica que el $88 \%$ desaprovecha las oportunidades que brindan los sistemas de información. Se destaca, además, la necesidad de implementar buenas prácticas para asegurar la seguridad de la información según las normas vigentes. Se aprobó la ejecución de un grupo de actividades para elevar en primer término la eficiencia del sistema informativo de la muestra de estudio, con vistas a continuar profundizando hacia la inteligencia empresarial.

\section{KEYWORDS ABSTRACT}

Systems of information

The necessity to maintain efficient systems of information in the prioritized companies of the territory of Pinar del Rio, Cuba, took us to carry out a diagnosis to determine the current state of the same ones and to propose group actions that congratulate the taking of decisions and the identification of the useful flows of information, to favor the organizational acting. The results of the study evidenced among other that alone $12 \%$ has an organized system of information and implemented according to the current requirements, what indicates that $88 \%$ is wasting the opportunities that offer the systems of information, also stands out the necessity to implement good practices to assure the security of the information according to the effective norms. The execution of a group of activities was approved to rise in first term the efficiency of the informative system of the study sample, with a view to continuing deepening toward the managerial intelligence.

Cuba

Recibido: 29 de noviembre de 2016 | Aceptado: 28 de febrero de 2017 | Publicado: 20 de abril de 2017 


\section{Introducción}

El desarrollo de las Tecnologías de la Información y las Comunicaciones (TIC) ha traído el surgimiento de los sistemas de información digital $y$, con ello, el uso de documentos electrónicos y en papel que, no en pocas ocasiones, con el exceso de duplicidades contribuyen a entorpecer la gestión de información en la empresa. A esto se debe agregar que, en la mayoría de los casos, existe un sistema informático con numerosas carpetas o directorios repletos de documentos en diversas formas, cada uno sustentado por una aplicación o un dispositivo distinto, sin criterio de organización, lo que dificulta la localización de los recursos informativos en el momento oportuno. Estos inconvenientes y las ventajas del almacenamiento digital estimulan el interés de los sistemas de gestión de información empresarial (Franganillo, 2009: p.400).

Se considera que la información es un recurso que se encuentra al mismo nivel que los recursos financieros, materiales y humanos, que hasta el momento habían constituido los ejes sobre los que había girado la gestión empresarial. Si la Teoría económica tradicional mantenía el capital, la tierra y el trabajo como elementos primarios de estudio, la información se ha convertido, ahora, en el cuarto recurso a gestionar (Muñoz Cañavate, 2003).

Desde el punto de vista de la gestión empresarial, el conocimiento del entorno, en un mundo cada vez más complejo y cambiante, origina una necesidad acuciante de información para la toma de decisiones, tanto para atacar nuevos mercados como para proteger a la empresa de agentes externos que puedan vulnerar su estabilidad.

Si una organización desea ser competitiva y permanecer en el tiempo, deberá identificar, crear, almacenar, transmitir y utilizar de forma eficiente la información y el conocimiento individual y colectivo de sus trabajadores, con el fin de resolver problemas, mejorar procesos o servicios y, sobre todo, para aprovechar nuevas oportunidades de negocio.

Paños Álvarez (2000) refiere que el saludable desempeño de las organizaciones de la sociedad actual está, en gran medida, determinado por su capacidad para crear valor por medio de la solución de los retos que le plantea el entorno, mediante la identificación de los flujos de información útiles que aporten valor a sus decisiones y al quehacer cotidiano de las mismas. Las empresas cubanas, al igual que las del resto del mundo globalizado, requieren mantener en unas y elevar en otras la competencia. Para ello es imprescindible identificar y mantener los flujos de información útiles que apoyen al cumplimiento de los objetivos de las organizaciones. En este sentido Caro, Fuentes y Soto (2013), sostienen:

Los Sistemas de Información existen en todo tipo de organizaciones y son fundamentales para que éstas puedan obtener, procesar, almacenar y gestionar su información. Se puede decir entonces, que el propósito de los Sistemas de Información es proveer datos e información a quien la necesite dentro de la organización a quien sirve (p. 54).

Por su parte, Bencomo (2012) agrega:

En el pasado como en el presente, los sistemas de información consisten en estándares establecidos para el procesamiento, almacenamiento y entrega de información a los miembros de la organización donde cada una de estas personas, requiere información distinta para la realización de su trabajo, en particular como instrumento en la toma de decisiones y en las actividades de control gerencial (p. 2) 
De las definiciones precedentes, se deduce que los autores sustentan los procesos de investigación consientes de la importancia de los sistemas de información para favorecer la toma de decisiones y el quehacer cotidiano de las organizaciones, que, según recomiendan los expertos, debe comenzar por "proporcionar la información necesaria a la alta dirección para la adecuada toma de decisiones" (Solana Álvarez, 2014).

En Ecuador, Quisimalin, De Esteban y Antonovica (2014) estudiaron desde el punto de vista gerencial el sistema de información de una empresa dedicada a la gestión de desechos sólidos, y refieren que el sistema de información empresarial es importante para la eficiencia operacional, la productividad, el servicio y la satisfacción de los clientes.

El desarrollo de la economía digital y el incremento del volumen mundial de información exigen de las empresas un reordenamiento de su sistema informativo, no quedando exentas de esto las empresas pinareñas.

El Perfeccionamiento Empresarial cubano es un sistema de dirección y gestión empresarial cuyo objetivo central es incrementar al máximo la eficiencia y competitividad de la empresa estatal socialista, otorgándosele las facultades y atribuciones necesarias para una correcta administración, con una concepción de avanzada en la gestión empresarial y las políticas, los principios y los procedimientos que propendan al desarrollo de la iniciativa, la creatividad y la responsabilidad de todos los jefes y los trabajadores. Constituye la guía y el instrumento de dirección para que las organizaciones empresariales puedan, de forma ordenada, realizar las transformaciones necesarias con el objetivo de lograr la máxima eficiencia y eficacia en su gestión. Por ello, el Estado cubano, no siendo ajeno a la función que desempeña el sistema de información en las empresas, realiza esfuerzos por reposicionar la gestión del componente informativo, sobre todo en las empresas en perfeccionamiento y fundamentalmente con el surgimiento de documentos normativos de distintas fuentes como son: el Comité Ejecutivo del Consejo de Ministros (2007), la Contraloría General de la República (2011), las normas ISO adoptadas por Cuba de la familia 30.000 para la gestión de archivos (Oficina Nacional de Normalización, 2013) y la ISO 27001 para la seguridad de la información (Oficina Nacional de Normalización, 2016). Esta última, tiene el objetivo de introducir buenas prácticas que sean acoplables a cualquier tipo de organización para gestionar la seguridad de la información y medir la eficacia del sistema.

El desarrollo de los sistemas de gestión ha llegado a los sistemas de información en las empresas que, más allá de la mera gestión de la información científico-técnica para actualizar al personal, trata de gestionar la información administrativa y también científico-tecnológica que facilita la toma de decisiones y permite el desempeño organizacional para el cumplimiento de sus objetivos.

En este sentido el desarrollo de las TIC ha permitido el diseño de diferentes softwares de cuadros de mando integral que permiten comunicar a todo el personal, el estado de cumplimiento de los objetivos a través de indicadores de desempeño.

\section{Según Goitia, Sáenz de Lacuesta y Bilbao (2008)}

La información está cobrando cada día una mayor importancia y el conocimiento es el motor de las organizaciones actuales. La empresa siempre ha considerado importante la información y ha tratado de interpretarla con visión de futuro, pero la globalización y el desarrollo de la red digital la han convertido en un recurso estratégico clave y ha hecho que las fuentes sean ilimitadas (p. 540). 
El desarrollo desigual de las empresas cubanas hace necesario establecer prioridades para lograr elevar la eficiencia de las mismas en función de los recursos.

El Ministerio de Ciencia, Tecnología y Medio Ambiente (CITMA) en Cuba, en virtud de las atribuciones conferidas por el acuerdo 4002 del Comité Ejecutivo del Consejo de Ministros (CECM) y lo establecido en el Decreto Ley No 252/2007 (Sistema de Dirección y Gestión Empresarial Cubano), Decreto No 281 (Reglamento para la Implementación y Consolidación del Sistema de Dirección y Gestión Empresarial Estatal), impulsa, favorece, orienta, evalúa y controla todo lo relativo a la Innovación, el Medioambiente, Calidad, la Información CientíficoTecnológica, la Propiedad Industrial y a sus Sistemas de Gestión en las empresas, en coordinación con el Grupo de Perfeccionamiento Empresarial (GEPE) del Ministerio de Economía y Planificación.

La Delegación territorial del Ministerio de Ciencia, Tecnología y Medio Ambiente (CITMA) en Pinar del Río, se dio a la tarea, de seleccionar a un grupo de empresas, de un total de noventa en la provincia en base a: la inserción en el perfeccionamiento empresarial que desarrolla el país, capacidades, la estructura y tradición en las actividades de información, la disponibilidad de recursos, la contribución a las exportaciones y sustitución de importaciones y el papel y lugar en la producción mercantil del territorio. De este análisis surgieron 25 empresas priorizadas por el CITMA en el territorio para reforzar su trabajo en la actividad de ciencia, tecnología e innovación, incluyéndose los sistemas de información como parte de los sistemas de gestión que se necesitan fortalecer.

\section{Objetivos de la investigación}

Objetivo general:

- Determinar el estado actual de los sistemas de información en las empresas priorizadas por la Delegación territorial del CITMA en Pinar del Río, en base a las normativas cubanas legales establecidas para los sistemas de información en las empresas y la norma ISO 27001 adoptada por Cuba.

Objetivos específicos:

- Identificar acciones que favorezcan el mejor desempeño organizacional y con ello la eficiencia del sistema de información de las empresas del territorio.

\section{Metodología}

Se realizó la identificación de las empresas priorizadas en el 2016 por el CITMA en el sistema de ciencia e innovación tecnológica.

En la investigación se emplearon distintos métodos y técnicas de recolección y análisis de información:

- De manera general se utilizó la técnica de diagnóstico para determinar el estado de los sistemas de información, teniendo en cuenta las exigencias actuales para el sector 
empresarial cubano, haciendo coincidir la muestra con la población (Calero, 1976).

- Método histórico-lógico: posibilitó analizar diferentes criterios y profundizar en la evolución, tendencias y generalizaciones del desarrollo de los sistemas de información.

- Método sociológico o empírico: comprende la observación, la revisión de documentos, encuestas y entrevistas, y fueron propuestos como vía para el diseño de las acciones previstas. La observación participante y las entrevistas semi-estructuradas se utilizaron con el objetivo de alcanzar mayor profundidad en el estudio de las potencialidades y debilidades que subyacen en el objeto de estudio. Las encuestas permitieron recolectar datos sobre el comportamiento de los sistemas de información en el desarrollo de los procesos de la gestión de información empresarial (el procesamiento de la encuesta se realizó a través del software informático estadístico SPSS). El análisis documental se utilizó para obtener datos acerca de cómo se comporta el proceso de la gestión de información en las empresas seleccionadas a través del sistema de información y cómo se planificaron, organizaron y ejecutaron las acciones para el mejoramiento del mismo. Se consultaron los documentos que rigen la política de información cubana y las normas vigentes.

- Se utilizó un abordaje cualitativo de investigación-acción participativa. Este es un método sistemático e intencional de indagación, que fue utilizado por los investigadores para reflexionar y actuar en conjunto con los especialistas de las empresas. La unión efectiva del conocimiento con la acción, amparada en la reflexión cíclica, tuvo el objetivo de convertirlos en coinvestigadores, para que, de esta forma, fueran capaces de tomar conciencia del por qué, cómo y para qué del proceso de la gestión de información en la organización, además de tener una actuación protagónica en la transformación de la situación imperante en cuanto a los sistemas de información en las empresas. El diagnóstico participativo se realizó a través de un Taller con el objetivo de determinar las expectativas de los asistentes respecto a la experiencia en la actividad, así como sus capacidades instaladas y deficiencias para lograr desarrollarla (tabla 1).

Tabla 1. Diagnóstico participativo

\begin{tabular}{||l||l||l||}
\hline \hline Actividad & Objetivos & Técnicas empleadas \\
\hline \hline Taller & $\begin{array}{l}\text { Encuadrar expectativas. } \\
\text { Analizar las diferencias entre } \\
\text { el diagnóstico tradicional y el } \\
\text { participativo. } \\
\text { Hacer conscientes a los } \\
\text { actores de las potencialidades } \\
\text { y deficiencias del sistema. }\end{array}$ & \\
\hline
\end{tabular}




\section{Resultados y Discusión}

El diagnóstico participativo fue una fase esencial en el proceso para la posterior construcción del plan de acciones para el sistema de información en la muestra de estudio. Al concluir se obtuvieron los siguientes resultados:

- Que los individuos objeto de investigación devienen sujetos de investigación, pues ellos mismos diagnosticaron los problemas existentes, sus deficiencias como gestores de información y sus capacidades instaladas que constituyen fortalezas.

- Manifiestan la falta de capacitación, fundamentalmente en la implementación de la Norma NC-ISO 27001.

- Se identifican las tecnologías instaladas que constituyen fortalezas para el sistema.

- Se desconocen las posibilidades de obtener recursos informativos y servicios especializados de inteligencia empresarial que puedan apoyar el sistema.

Del diagnóstico realizado sobre el Sistema de Información a las 25 empresas actualmente priorizadas por el CITMA en Pinar del Río, se desprendió que el 100\% de las mismas utilizan software(s) y alguna base de datos para su gestión de información; estos mayormente han sido implantados por exigencias de su organismo superior, no por reconocerse la necesidad y las bondades de estas tecnologías.

En la figura 1 se muestra que solo cuatro empresas (16\%) tienen identificados y documentados los flujos de información por procesos, que tres de ellas (12\%) refieren tener documentada la tipología de información de su organización. En este sentido, los autores de la presente investigación, de acuerdo con Paños Álvarez (2000), ratifican la necesidad de identificar los flujos útiles de información que favorezcan a la toma de decisiones y el mejor desempeño de la organización, como así también la necesidad de eliminar las duplicidades innecesarias; controlando además a través de la tipología, el origen, el destino y las fuentes de donde proviene la información, el período de conservación y acceso a la misma.

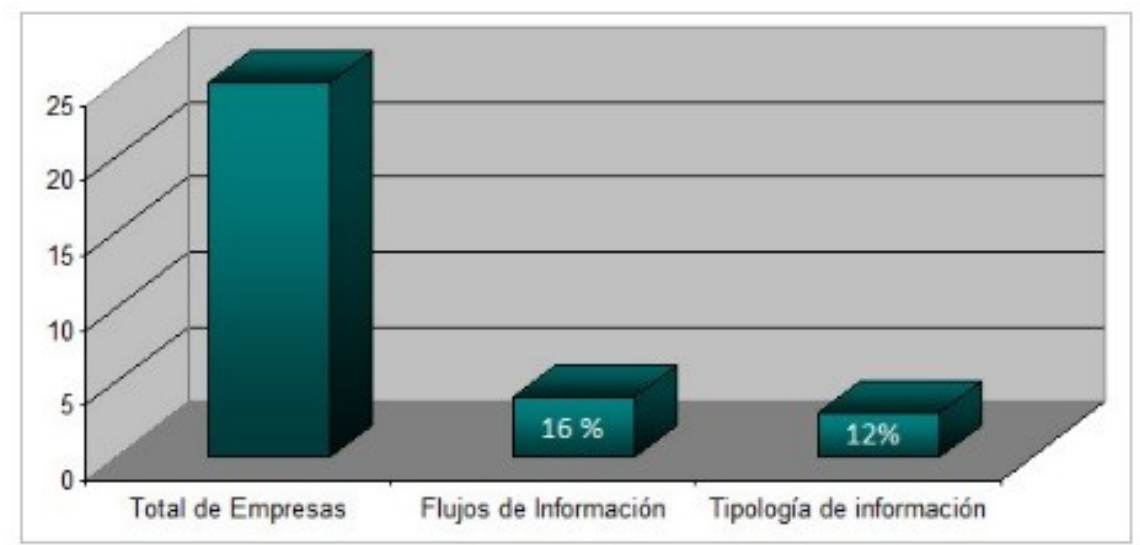

Figura 1. Flujos de información y Tipología. 
En cuanto al uso de las tecnologías como medios de comunicación, los resultados arrojaron que solo cinco empresas $(20 \%)$ tienen Intranet y quince $(60 \%)$ utilizan página web. Es a partir de estos datos que se considera bajo el uso de estas herramientas en el primer indicador (intranet), para favorecer la gestión del conocimiento de los miembros de la organización, y en el segundo indicador (página web), teniendo en cuenta que estamos en la era de la informatización, divulgar los resultados de su gestión es fundamental para el desarrollo de todas las organizaciones (figura 2).

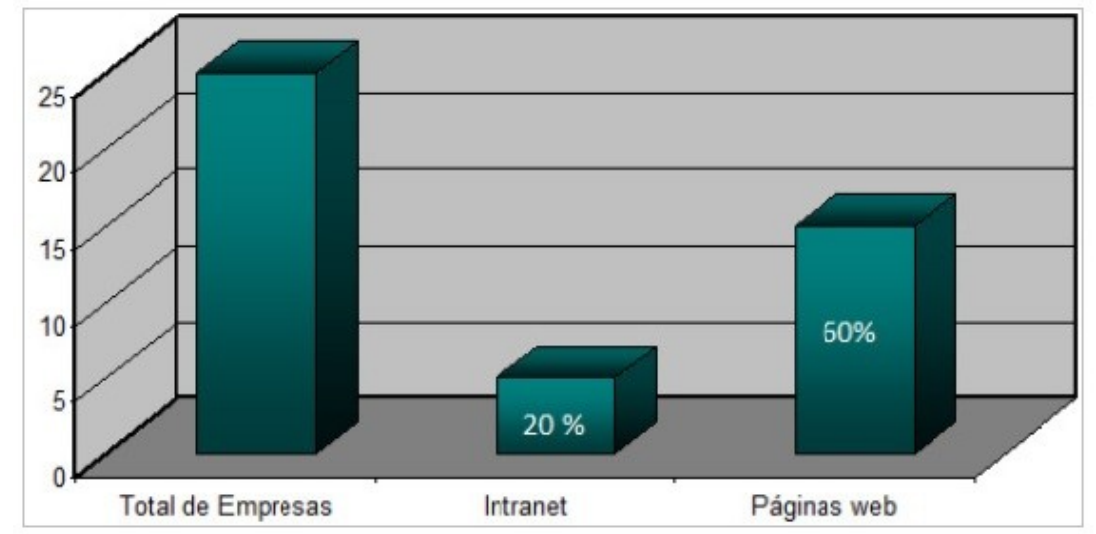

Figura 2. Uso de las tecnologías como medio de comunicación.

Según la organización Gemus (2016), los cuadros de mando integral constituyen valiosas herramientas que gestionan la información hacia adentro de la organización y permiten medir el cumplimiento de la estrategia empresarial así como la eficacia de los procesos internos. En este sentido, la figura 3 muestra que solo tres empresas (12\%) tienen implementado algún cuadro de mando integral, lo que indica un bajo nivel de gestión de información teniendo en cuenta las bondades de esta tecnología.

Dado que las empresas no se encuentran totalmente conectadas a Internet, se sugiere para la gestión de información externa utilizar las intranet, insertando el resultado de servicios especializados de análisis de información como vigilancia tecnológica y estudios de tendencia, contratados por la empresa a centros de información que cuentan con personal entendido en estos temas para lograr una eficiente gestión de información empresarial.

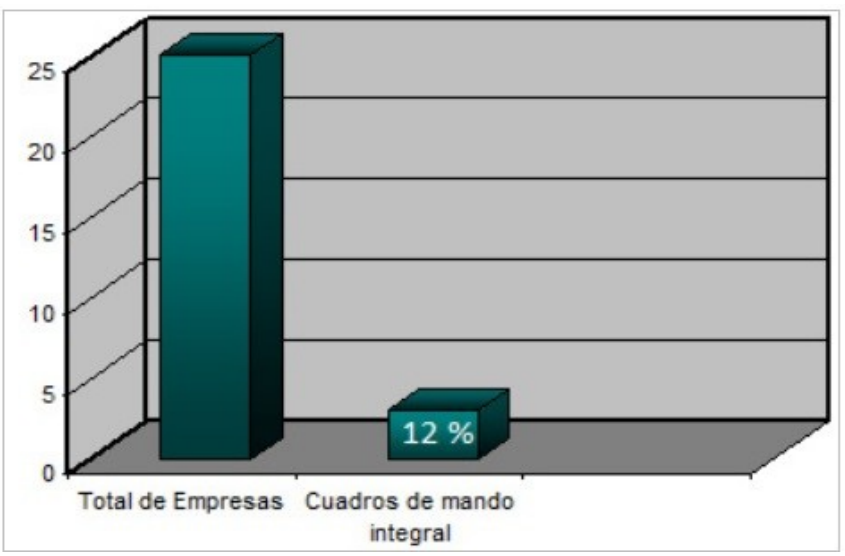

Figura 3. Uso de Cuadros de mando integral en la gestión. 
De la totalidad, solo el $12 \%$ de las empresas priorizadas por el CITMA en el territorio de Pinar del Río tienen un Sistema de Información organizado e implementado según algunas de las normativas y los requerimientos actuales que se exigen en el país. De esto se desprende que persisten directivos que desaprovechan las oportunidades que brindan los sistemas de información (88\%).

La norma internacional ISO 27001:2016, adoptada por Cuba para dar garantía a la seguridad de la información, no se ha podido implementar y certificar en el territorio en estudio. Este hecho destaca que los sistemas de información de las empresas se encuentran en riesgo. Según datos publicados por la Oficina Nacional de Normalización, actualizados en febrero de 2017, en el territorio de Pinar del Río existen 18 empresas que han certificado algunos de los sistemas de gestión. Sin embargo aún en el país ninguna ha logrado certificar el Sistema de Gestión de la Seguridad de la Información (figura 4), si bien existe la posibilidad de hacerlo, ya que para los clientes constituye una garantía de calidad y confidencialidad y, para la propia organización, esta certificación permite ofrecer una imagen favorable tanto nacional e internacional, ya que implementa en sus procesos normas de buenas prácticas reconocidas para asegurar la seguridad de los activos de información.

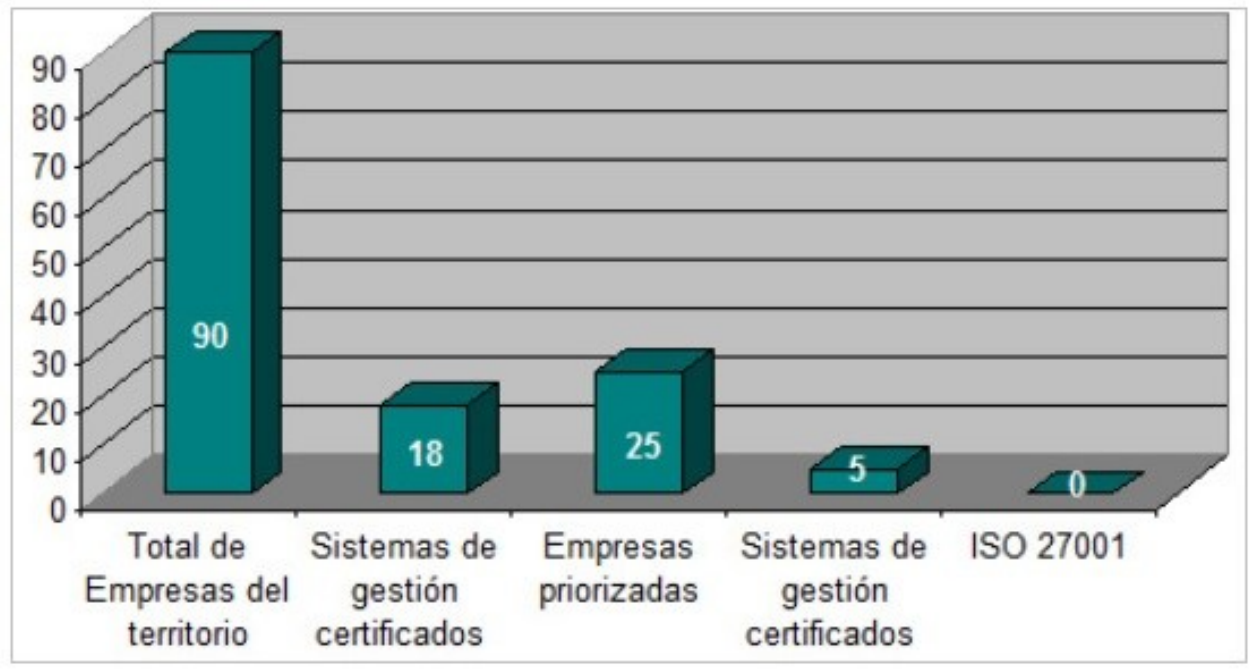

Figura 4. Resultados de la certificación de los sistemas de gestión.

Muy pocas empresas del territorio utilizan la vigilancia tecnológica y los estudios de tendencias como herramientas que les permitan mantenerse competitivas y actualizadas en vista a la posible introducción de nuevas y mejoradas tecnologías.

Todos estos resultados llevaron a reflexionar sobre la necesidad de una evaluación en conjunto con las empresas, a proponer al Consejo Técnico Asesor provincial un plan de acciones (tabla 2) para mitigar estos resultados en función de realizar mejoras en la gestión de información de las empresas estudiadas. 
Palabra Clave (La Plata), abril 2017, vol. 6, n² 2, e022. ISSN 1853-9912

Tabla 2. Plan de acciones para la implementación de los Sistemas de Información en las empresas priorizadas.

\begin{tabular}{|c|c|c|c|c|}
\hline No. & Actividades & Responsable & Participa & Plazo \\
\hline 1 & $\begin{array}{l}\text { Curso de Sistemas de } \\
\text { Información }\end{array}$ & CIGET & $\begin{array}{l}\text { Especialistas de } \\
\text { Empresas y CIGET }\end{array}$ & $\begin{array}{l}\text { 10-13 enero } \\
2017\end{array}$ \\
\hline 2 & $\begin{array}{c}\text { Entrega de documentación } \\
\text { técnica para el desarrollo del } \\
\text { sistema }\end{array}$ & ClGET & $\begin{array}{c}\text { Especialistas de } \\
\text { Empresas y CIGET }\end{array}$ & $\begin{array}{l}\text { 10-13 enero } \\
2017\end{array}$ \\
\hline 3 & Desarrollo del sistema & Empresas & $\begin{array}{l}\text { Especialistas de } \\
\text { Empresas y CIGET }\end{array}$ & $\begin{array}{c}\text { Enero- } \\
\text { Noviembre }\end{array}$ \\
\hline 4 & $\begin{array}{l}\text { Documentar los flujos de } \\
\text { información }\end{array}$ & $\begin{array}{l}\text { Especialista de } \\
\text { Ciencia y } \\
\text { Técnica }\end{array}$ & $\begin{array}{l}\text { Especialistas de } \\
\text { Empresas y CIGET }\end{array}$ & $\begin{array}{l}16 \text { enero al } 28 \\
\text { febrero }\end{array}$ \\
\hline 5 & $\begin{array}{l}\text { Documentar tipologia de } \\
\text { información }\end{array}$ & $\begin{array}{l}\text { Especialista de } \\
\text { Ciencia y } \\
\text { Técnica }\end{array}$ & $\begin{array}{l}\text { Especialistas de } \\
\text { Empresas y CIGET }\end{array}$ & $\begin{array}{l}16 \text { enero al } 28 \\
\text { febrero }\end{array}$ \\
\hline 6 & $\begin{array}{c}\text { Propuesta de contenidos para } \\
\text { soporte del sistema }\end{array}$ & $\begin{array}{c}\text { Especialista de } \\
\text { Ciencia y } \\
\text { Técnica }\end{array}$ & $\begin{array}{c}\text { Especialistas de } \\
\text { Empresas y } \\
\text { Especialista } \\
\text { Información CIGET }\end{array}$ & $1-3$ marzo \\
\hline 7 & $\begin{array}{l}\text { Diseño de productos y } \\
\text { servicios para el sistema }\end{array}$ & Empresas & $\begin{array}{l}\text { Especialistas de } \\
\text { Empresas y CIGET }\end{array}$ & $\begin{array}{c}\text { Enero- } \\
\text { Noviembre } \\
\end{array}$ \\
\hline 8 & Instalación de software Diriger & $\begin{array}{l}\text { Especialista de } \\
\text { C. y T. }\end{array}$ & Informático y ClGET & $6-24$ marzo \\
\hline 9 & $\begin{array}{c}\text { Diseño de Intranet y página } \\
\text { web }\end{array}$ & $\begin{array}{c}\text { Especialista } \\
\text { Informático de } \\
\text { Empresa }\end{array}$ & $\begin{array}{l}\text { Informático CIGET y } \\
\text { Especialistas de } \\
\text { Información }\end{array}$ & 13 marzo a 30 \\
\hline 10 & Revisión de la documentación & $\begin{array}{l}\text { Esp. Inform. } \\
\text { CIGET }\end{array}$ & $\begin{array}{c}\text { Especialistas C y T. } \\
\text { empresa }\end{array}$ & $\begin{array}{l}\text { Marzo, Julio, } \\
\text { septiembre, } \\
\text { noviembre }\end{array}$ \\
\hline 11 & Presentación a certificación & Empresa & CIGET y Empresa & $\begin{array}{l}\text { Diciembre } \\
2017\end{array}$ \\
\hline
\end{tabular}

El Plan de acciones aprobado por el Consejo Técnico Asesor comenzó a ejecutarse en el mes de enero de 2017 con la capacitación y la entrega de la documentación a las empresas de estudio. El diagnóstico participativo motivó la ejecución de las actividades, dado que el $100 \%$ de la muestra fue capacitada representando hasta la fecha un $18 \%$ de ejecución del plan previsto.

Los autores de la presente investigación coinciden con los autores reportados en que el sistema de información empresarial es importante para la eficiencia operacional, la productividad y la satisfacción de los clientes. Consideran, además, coincidiendo en este caso con Goitia, Sáenz-de-Lacuesta y Bilbao (2008), que las empresas deben asumir una dinámica de eficiencia en su sistema informativo y una vigilancia sobre el entorno en el cual se desarrollan, de manera tal que les permitan recoger, filtrar y analizar la información como elemento esencial con vistas a implementar la inteligencia empresarial.

\section{Conclusiones}

Los resultados del diagnóstico muestran que en la mayoría de las empresas constatadas en el estudio se requiere continuar actuando para elevar la eficiencia de los sistemas de información. Tal es así con la implementación de la norma internacional 27001 adoptada por Cuba, la cual constituye una práctica muy necesaria para garantizar la competitividad de las organizaciones y demostrar la seguridad de la información a los clientes y demás partes interesadas.

El Plan de actividades diseñado y aprobado abarca desde la capacitación del personal y la implementación de las acciones, hasta la certificación del sistema de seguridad de la información de las organizaciones estudiadas. Este Plan constituye una fortaleza para elevar la 
eficiencia del sistema de información de la muestra de estudio, con vistas a profundizar la inteligencia empresarial.

\section{Referencias bibliográficas}

Bencomo F. F. (2012). Los sistemas de información como atractores en las organizaciones desde una visión compleja. Observatorio de la Economía Latinoamericana, 162. 4p.Recuperado de: http://www.eumed.net/cursecon/ecolat/ve/2012/fjbf.html

Calero Vinela, A. (1976). Técnicas de muestreo. La Habana: Editorial Pueblo y Educación.

Caro, A.; Fuentes, A. y Soto, A. (2013). Desarrollando sistemas de información centrados en la calidad de datos. Ingeniare. Revista chilena de ingeniería, 21(1), 54-69. Recuperado de: http://www.scielo.cl/scielo.php?script=sci arttext\&pid=S0718-33052013000100006

Comité Ejecutivo del Consejo de Ministros (2007). Decreto Ley 281. Reglamento para la implantación y consolidación del sistema de dirección y gestión empresarial estatal. Capítulo XVII Sistema Informativo. La Habana, Cuba.

Contraloría General de la República (2011). Resolución 60. Normas del Sistema de Control Interno. Secciones tercera y cuarta. La Habana, Cuba.

Franganillo, J. (2009). Gestión de información personal: elementos, actividades e integración. El profesional de la información, 18(4), 399-406. DOI: 10.3145/epi.2009.jul.06 Recuperado de: http://www.elprofesionaldelainformacion.com/contenidos/2009/julio/index.html

Gemus (2016). Forma de gestión no estatal y su producto lider Diriger. En: Centro de Información y Gestión Tecnológica, CIGET Pinar del Río. Boletín Gestión Empresarial (2), 4-6.

Goitia, S.; Sáenz-de-Lacuesta, S.; Bilbao, M. (2008). Implantación de sistemas de información empresarial. El profesional de la información, 17(5), 540-545. Recuperado de: http://www.elprofesionaldelainformacion.com/contenidos/2008/septiembre/08.pdf

Ministerio de Justicia. (2011). Decreto Ley 281. Gaceta Oficial de la República de Cuba, 010 (Extraordinaria).

Muñoz Cañavate, A. (2003). Sistemas de información en las empresas. Hipertext.net, 1 Recuperado de: https://www.upf.edu/hipertextnet/numero-1/sistem_infor.html

Oficina Nacional de Normalización (2013). NC-ISO 30302 Sistemas de gestión para los documentos. Guía para la implementación.

Oficina Nacional de Normalización (2016). NC-ISO/IEC 27001. Tecnología de la Información. Técnicas de Seguridad. Sistemas de Gestión de la Seguridad de la Información. Requisitos. 41p.

Oficina Nacional de Normalización (2017). Base de datos de sistemas de gestión certificado. Recuperado de: http://www.nc.cubaindustria.cu/certificacion.html

Paños Álvarez, A. (2000). Reflexiones sobre el papel de la información como recurso competitivo de la empresa. An Doc, (2), 21-38. Recuperado de: http://eprints.rclis.org/11935/1/AD02-1999.PDF. 
Quisimalin, M.; de Esteban Curiel, J.; Antonovica, A. (2014). Percepción gerencial del sistema de información empresarial en la problemática ambiental de Ecuador. Observatorio Medioambiental, $17 . \quad$ p. 325-340. Recuperado de: http://revistas.ucm.es/index.php/OBMD/article/view/47196

Solana Álvarez, J. M. (2014). El sistema de información de una organización. Necesidad de implicación de la dirección. Anuario Jurídico y Económico Escurialense, XLVII. Recuperado de: http://www.rcumariacristina.net:8080/ojs/index.php/AJEE/article/view/202/169 